\title{
Synchrotron radiation photoionization mass spectrometry of laser ablated species
}

\author{
J. Alvarez Ruizz, ${ }^{\mathrm{a}, *}$, A. Casu ${ }^{\mathrm{b}}$, M. Coreno ${ }^{\mathrm{c}}$, M. de Simone ${ }^{\mathrm{d}}$, L.M. Hoyos Campo ${ }^{\mathrm{e}}$, A.M. Juarez-Reyes ${ }^{\mathrm{e}}$, \\ A. Kivimäki ${ }^{\text {d }}$, S. Orlando ${ }^{c}$, M. Sanz ${ }^{\text {a }}$, C. Spezzani ${ }^{\text {f }}$, M. Stankiewicz ${ }^{g}$, D.M. Trucchi ${ }^{\text {h }}$ \\ a Instituto de Química Física Rocasolano,CSIC, 28006 Madrid (E), Spain \\ ${ }^{\mathrm{b}}$ University of Cagliari, 09042 Monserrato (I), Italy \\ ${ }^{\text {c } C N R-I M I P, ~ c / o ~ L a b . ~ E l e t t r a ~ T r i e s t e, ~} 00016$ Montelibretti (I) Italy \\ d CNR-INFM, Laboratorio Nazionale TASC, 34149 Trieste, Italy \\ e ICF-UNAM Cuernavaca (Mx), Mexico \\ ${ }^{\mathrm{f}}$ Sincrotrone Trieste, 34149 Trieste (I), Italy \\ g Jagiellonian University, 30-059 Kraków (Pl), Poland \\ ${ }^{\mathrm{h}}$ CNR - ISC, 00016 Montelibretti (I), Italy
}

\section{A R T I C L E I N F O}

\section{Article history:}

Received 4 June 2009

Received in revised form 28 July 2009

Available online 1 October 2009

\section{Keywords:}

Clusters

Laser

Ablation

Synchrotron

TOF-MS

Sulfur

\begin{abstract}
A B S T R A C T
The present paper describes an experimental apparatus suitable to create and study free clusters by combining laser ablation and synchrotron radiation. First tests on sulfur samples, S, showed the production, through laser ablation, of neutral $S_{n}$ clusters $(n=1-8)$. These clusters were ionized using synchrotron radiation at photon energies from $160 \mathrm{eV}$ to $175 \mathrm{eV}$, across the $\mathrm{S} 2 \mathrm{p}$ core edge. The feasibility of such combined ablation-synchrotron radiation experiments is demonstrated, opening new possibilities on the investigation of free clusters and radicals.
\end{abstract}

(C) 2009 Elsevier B.V. All rights reserved.

\section{Introduction}

Tuneable excitation energy and high photon flux turn thirdgeneration synchrotron radiation sources into a formidable and unique tool for investigations on the interaction between light and matter. Synchrotron radiation facilities have allowed great advances in atomic and molecular physics during the last decades and this is also becoming true for research on free atomic and molecular clusters [1,2].

Clusters drive interest and expectation because they represent the intermediate stage between isolated species (molecules or atoms) and the solid state. Moreover, clusters are one of the foundations of nanotechnology. To date, the majority of investigations about clusters using synchrotron radiation have focused on their solid-bound form, i.e., as deposits on surfaces. Fewer studies have dealt with their properties as free entities in their gas phase form. An advantage of studying clusters in the gas phase is that one can use experimental techniques that have been well established in atomic and molecular spectroscopy. In those studies, gas phase

\footnotetext{
* Corresponding author. Tel.: +34 915619400; fax: +34 915642431

E-mail address: j.alvarez@iqfr.csic.es (J. Alvarez Ruiz).
}

clusters are generally created through adiabatic expansion of high pressure samples [3] or in plasma cluster sources [4].

Laser ablation is extensively used across a diverse range of disciplines, including those involving the production of new materials, clusters, and thin film growth (Pulsed Laser Deposition). Consequently, diagnostics of laser produced plasmas have always been an important topic of research with the aim of tailoring material properties controlling the concentration and evolution of different species present in the plume. Some of the authors of the present work investigate the physics and the dynamics of the laser ablation processes in their home laboratories, placing special attention to the creation and properties of clusters inside the plume [5-7]. To our knowledge, synchrotron-based studies of laser-ablated clusters have just recently been reported [8-12]. However, none of them has dealt with the systematic study of innershell photoionization and its cluster size dependence.

The goal of the present work is to present a set-up that combines, in an experimentally simple apparatus, the production of clusters with their simultaneous study in gas phase using synchrotron radiation. In our arrangement, clusters are produced by laser ablation of a selected target inside a vacuum chamber which is attached as an end-station to a synchrotron radiation beamline. In the experiments reported here, the photoionization yield and frag- 
mentation of $S_{n}$ clusters at the sulfur $S 2 p$ core edge was measured using a time-of-flight mass spectrometer (TOF-MS).

\section{Description of the apparatus}

The experimental apparatus is schematized in Fig. 1. It consists of a multiport stainless steel chamber which houses the sample, a TOF-MS, the pumps and vacuum gauges and also several free ports for the entrance of the laser and synchrotron light and viewing purposes.

Any flat solid sample can be employed as a target for laser ablation. Samples are mounted in a rotatable metallic holder plugged to a 3D translational stage for positioning and alignment purposes. The target is placed within the collecting plates of the TOF-MS and close to the interaction region. The interaction region is defined by the perpendicular crossing of the synchrotron radiation with the centre of the plates corresponding to the extracting region of the TOF-MS. The 3D translational stage together with the holder are designed so that they can be decoupled from the main vacuum chamber and pumped down independently. Thus, the replacement of the sample can be done without breaking the vacuum of the main chamber. Carbon tape is used to glue the sample to the holder so that the target was electrically grounded through the positioning system.

Opposite to the sample entrance in the main chamber there is a window through which laser pulses are sent for ablation normal to the target surface. Ablation is carried out by an external pulsed laser, conveniently focussed to achieve the laser fluence conditions necessary to ablate the target. In the present studies, a commercial Nd:YAG laser with a second harmonic module was used for ablation. This laser yielded $10 \mathrm{~ns}$ pulses of $532 \mathrm{~nm}$ photons with an energy of $6.7 \mathrm{~mJ}$ per pulse, working at a repetition rate of $10 \mathrm{~Hz}$.

The TOF-MS is custom made and optimized for the extraction of positive ions in ablation experiments. The total length of the TOF spectrometer is $593 \mathrm{~mm}$ and the detector used is a $18 \mathrm{~mm}$ diameter commercial Galileo channeltron. The extraction stage of the TOF-MS consists of two circular plates ( $80 \mathrm{~mm}$ external diameter) with an inner diameter hole of $15 \mathrm{~mm}$. The admittance holes are covered by a mesh to create a homogeneous field. The potentials for positive ion collection use here corresponded to $+200 \mathrm{~V}$ and $-200 \mathrm{~V}$ for the repelling and accelerating plate, respectively. After the extraction stage, there is an accelerating stage consisting of six

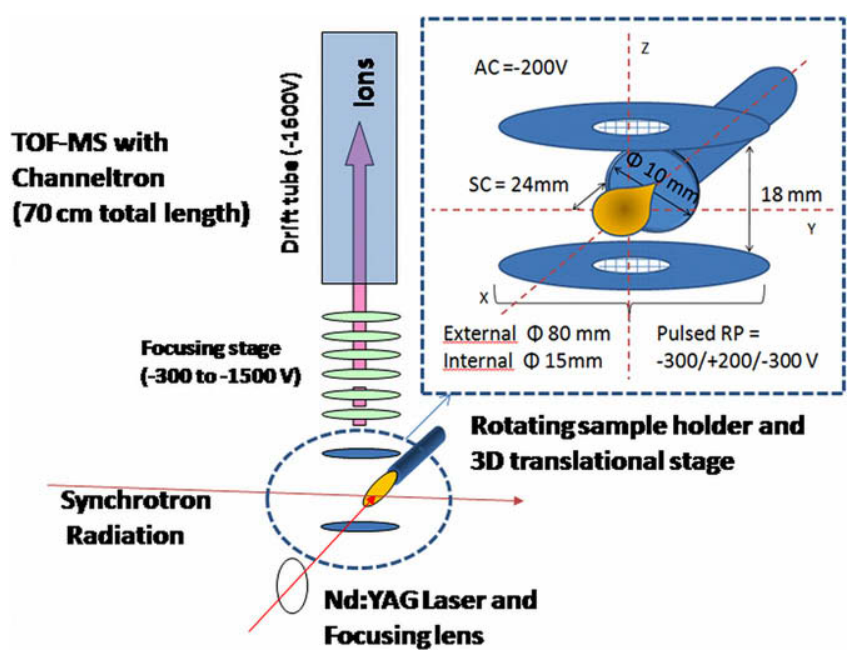

Fig. 1. Scheme of the apparatus to laser ablate a solid sample and to detect ionized species by the synchrotron light. The inset shows a detailed picture of the interaction region and lengths (AC - accelerating plate; $\mathrm{RP}$ - repelling plate; SC source to centre distance). circular plates separated $13 \mathrm{~mm}$ from each other. Each of these plates presents an internal hole of $35 \mathrm{~mm}$. These six plates are connected by a chain of resistors so that the created field varied smoothly from $-300 \mathrm{~V}$ (TOF outermost plate) to $-1500 \mathrm{~V}$. The TOF drift tube is connected to a voltage of $-1600 \mathrm{~V}$. The channeltron is operated at a voltage of $-3300 \mathrm{~V}$ and the collected pulses, corresponding to our signal are sent to an oscilloscope or to an ACAM card for data recording.

The vacuum chamber is designed to be mounted as an end-station of a synchrotron radiation beamline. The frame holding the chamber allows the alignment of the station so that the synchrotron light crosses the ablation plume just in the centre of the extraction plates of the TOF-MS. Synchrotron radiation and plume expansion are perpendicular and both normal to the TOF-MS axis. The alignment of the target-laser-plume-synchrotron radiationTOF axis is crucial for the optimization of ionization and collection efficiency.

Turbo pumps and vacuum gauges are attached to the chamber to control and monitor the vacuum. Base pressures below $10^{-6}$ mbar are used for the proper functioning of the TOF-MS and the safe operation of the synchrotron beamline.

It is worth mentioning that, although the present configuration is used for the collection of ions, many spare ports are available for the inclusion of other detectors for spectroscopy. In particular, a photon or an electron detector can be mounted under the TOFMS for photon induced fluorescence spectroscopy or photoelectron spectroscopy, as well as for coincidence experiments with ions.

The current ion collection scheme does not initially discriminate between the ions directly produced in the ablation and the ones ionized by the synchrotron light. In order to remove the unwanted direct ions, the repelling plate of the TOF-MS is initially set to a negative voltage (more negative than the one applied to the accelerating plate) which sweeps away all the positive direct ions from the interaction region. Depending on the applied voltage and amount of ablated particles, cations are typically removed in

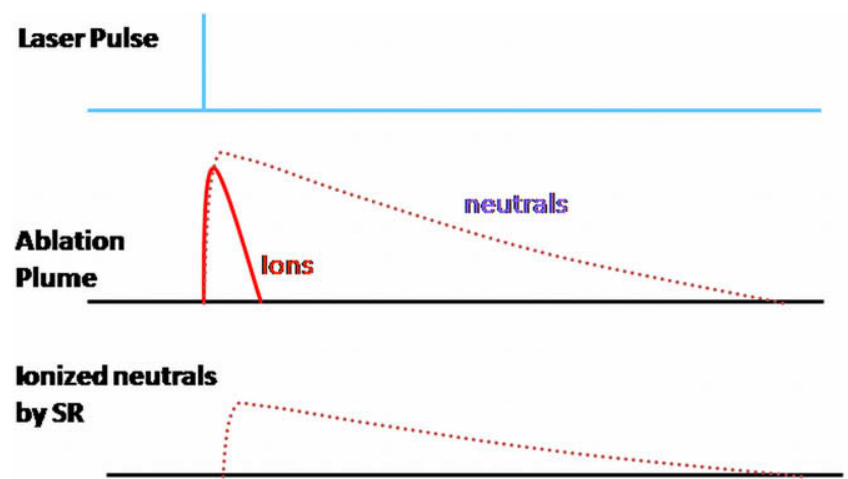

Potentials

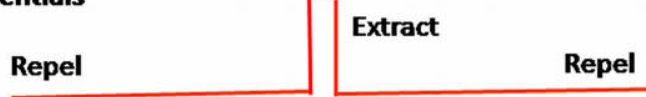

TOF window

Start of acquisition

Time ( $\mu s)$

\begin{tabular}{llllllllllll}
\hline & & & & & & & & & & & \\
0 & 2 & 4 & 6 & 8 & 10 & 12 & 14 & 16 & 18 & 20
\end{tabular}

Fig. 2. Summary of the description of the timing of the experiment. 
less than $5 \mu$ s after the ablation pulse. Once the direct ions are eliminated, the repelling plate is set back to a potential of $+200 \mathrm{~V}$ to collect the ionized clusters produced by the synchrotron light. However, there is one more problem to overcome before we can record a resolvable TOF mass spectrum. Due to the time structure of the storage ring of the synchrotron, which consists of a train of picosecond pulses separated by a few nanoseconds, the synchrotron light acts as a continuous light source and neutral clusters are ionized constantly. As the duration of an ablation plume can be of milliseconds, ions will arrive to the TOF detector at any time, yielding a useless TOF mass spectrum. In order to overcome this limitation, we collect the ionized species in a pulsed fashion. This is achieved by switching the potential of the repelling plate from the extraction conditions $(+200 \mathrm{~V})$ back to a negative voltage. According to simulations and experimental results, the extracting potential needs to be applied for about $1-1.5 \mu$ s (for the current voltages) in order to allow the ionized species to enter the TOFMS. This pulsed potential, which is applied to the repelling plate is achieved using a commercial High Voltage switch from Behlke (model GHTS 60). This voltage switch is synchronized with the laser ablation using the pulse of a fast photodiode that detects a reflection of the laser light and triggers the ion collection. The time description of the experiment is summarized in Fig. 2. As it will be shown in the results, the mass resolutions of the TOF-MS is limited by the duration of the extracting pulse.

\section{Experiments}

The experiment presented here was carried out at the Gas Phase Photoemission beamline [13] at the Elettra synchrotron radiation laboratory in Trieste (Italy). The electron storage ring provides synchrotron radiation pulses ( $\sim 70$ ps duration) every 2 ns. The beamline is capable of operating in the photon energy range of 15$1000 \mathrm{eV}$. The incoming radiation produced at an undulator in the beamline is monochromatized by a spherical grating monochromator equipped with a movable planar premirror. Five different gratings can be used depending on the photon energy region of interest. The pulses arriving at the interaction region carried around 2000 photons in average spread on an area of $0.01 \mathrm{~mm}^{2}$.

These first experiments were conducted on pressed powder $S$ pellets. A sulfur sample was selected since it had already been investigated with a more conventional vaporization method [14] and because it was a good starting point for future studies of sulfur containing systems like CdS or other chalcogenic semiconductors in general. The $S$ pellets were ablated by the second harmonic pulses of an Nd:Yag laser $(532 \mathrm{~nm})$ operated at $10 \mathrm{~Hz}$. The laser pulses were focused by an external lens $(60 \mathrm{~cm}$ focal distance) at $5 \mathrm{~cm}$ behind the target surface. Placing the target out of focus allowed us to work with a moderate ablation rate which reduced space charging effects and facilitated the removal of direct ions. Taking into account these considerations, the used fluence was $1 \mathrm{~J} / \mathrm{cm}^{2}$ and the irradiated surface of the target was around $0.01 \mathrm{~mm}^{2}$.

The sulfur pellet was situated $2.4 \mathrm{~cm}$ away from the centre of the extracting fields of the TOF and the synchrotron light path. That distance was a compromise between the plume density encountered by the synchrotron light (the shorter the distance, the higher the interaction rate) and the time required to remove the direct ions from the collecting region (the longer the distance, the better removal of direct ions). It is worth mentioning that the presence of the metallic holder worsened the extraction efficiency of the TOFMS when the sample was brought closer to the centre of the plates. A compromise between plume density and extraction efficiency determined the optimal position of the metallic holder of the sulfur pellet.

As it was described above, the potential of the TOF repelling plate was initially set to a negative voltage larger than the accelerating plate $(-300 \mathrm{~V}$ for the former; $-200 \mathrm{~V}$ for the latter) to remove the direct positive ions produced in the ablation so that only neutral species remained in the interaction region to get excited with the synchrotron light. Then, after some microseconds, the potential on the repelling plate was set to the TOF extracting configuration with a positive value of $+200 \mathrm{~V}$ (the applied extracting field in the ionization region was about $225 \mathrm{~V} / \mathrm{cm}$ ). Typically $5 \mu$ s were enough to remove all direct ions and give time for the

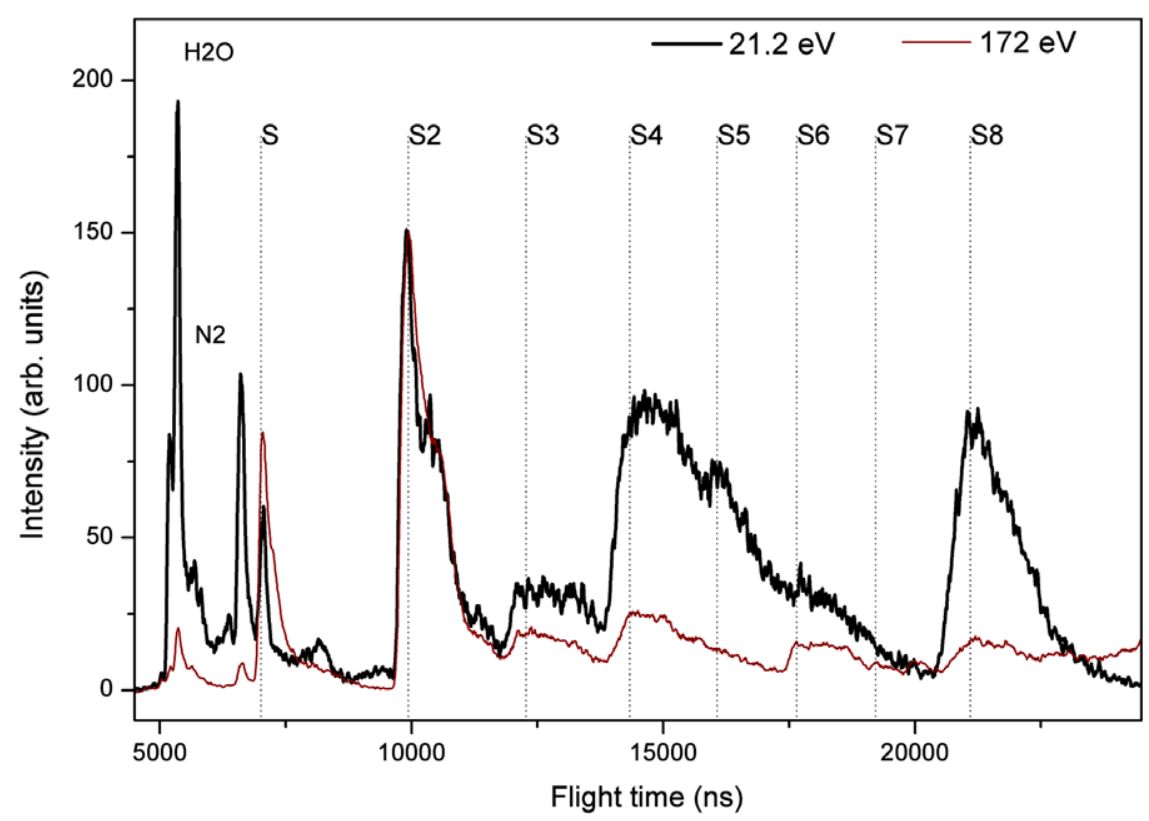

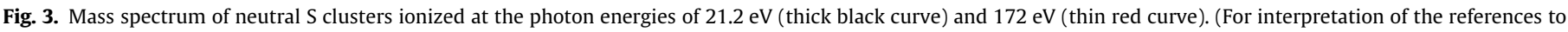
colour in this figure legend, the reader is referred to the web version of this article.) 
neutral species to fly to the centre of the TOF and interact with the synchrotron light. The extraction potential was applied to the TOF repelling plate only for about $1-1.5 \mu$ s to extract the neutral particles ionized by the synchrotron light and then it was set back to $-300 \mathrm{~V}$ to remove future ions.

With this short collecting time, the number of detected ions per laser pulse was quite low, yielding in average 0.5 ions per laser shot. In order to build up a mass spectrum with good statistics, acquisitions of 5-10 min were required. At a given photon energy, series of 600-1200 shots were acquired and summed up to construct a time-of-flight mass spectrum such as the ones presented in Fig. 3. The mass resolution featured by the TOF-MS was rather poor under the experimental conditions $(m / \Delta m<10)$ but was good enough to identify different S clusters up to $n=8$. The assignment of the mass spectrum was done using the SIMION simulation software (version 8).

\section{Results and discussion}

The mass spectra of synchrotron radiation ionized $S_{n}$ clusters have previously been reported by Teodorescu et al. [15]. They produced the clusters by heating a solid sulfur sample and ionized the vapor using different photon energies, concentrating particularly at the $S$ 2p edge. Comparing their ionization mass spectra with our measurement at valence ionization energies, we observe that the relative intensity of some species differs from one spectrum to the other (Fig. 3 shows our mass spectrum at $21.2 \mathrm{eV}$ ). Taking the $\mathrm{S}_{8}{ }^{+}$ion as the reference, the $\mathrm{S}_{2}{ }^{+}$and $\mathrm{S}_{4}{ }^{+}$peaks are clearly more intense in our experiment. Most likely, these differences reflect the variations in the production mechanism of the neutral S clusters (on one hand, pure thermal heating; on the other hand, laser ablation) and/or in the experimental conditions (temperature and laser power for heating and laser ablation, respectively). However, above the $S 2 p$ ionization limit, the mass spectra of Teodorescu et al. and our spectrum (Fig. 3 ) are quite similar. This apparent contradiction is explained by the fact that excitation/ionization of core electrons tends to dissociate the parent clusters, increasing the observation of small ionic fragments in detriment of heavier ones. This process "homogenizes" the differences in cluster production observed at valence (see latter discussion).

Laser ablation also produces direct positive and negative cluster ions [16,17]. Using low laser powers, Hearley et al. [16] observed $\mathrm{S}_{n}{ }^{+}$and $\mathrm{S}_{\mathrm{n}}{ }^{-}$clusters with $n$ up 15 and 10, respectively. Direct positively charged clusters were removed from the interaction region in our experiment before detection, while negatively charged clusters were not observed under the present conditions.

The kind of mass spectra presented in Fig. 3 were acquired at incident photon energies across the $S 2 p$ edge with the aim to investigate the partial ion yield of the detected clusters vs excitation energy. The photon energy was scanned from 160 to $175 \mathrm{eV}$ in steps of $0.25 \mathrm{eV}$ with a photon energy resolution of about $0.2 \mathrm{eV}$. The signal from $\mathrm{S}^{+}, \mathrm{S}_{2}{ }^{+}, \mathrm{S}_{3}{ }^{+}, \mathrm{S}_{4}{ }^{+}, \mathrm{S}_{6}{ }^{+}$and $\mathrm{S}_{8}{ }^{+}$of the obtained mass spectra was integrated and plotted versus excitation energy (Fig. 4). The energy region can be divided into three parts. In the first region, below $162.5 \mathrm{eV}$, only valence ionization processes are possible. Their probability does not change appreciably with photon energy close to the $2 \mathrm{p}$ edge. In the second region, at energies $162.5-170.5 \mathrm{eV}$, an S 2p inner-shell electron can be excited to unoccupied orbitals. The resulting core-excited states are short lived and typically decay via electron emission (so-called resonant Auger decay) to valence-excited final states. The main part of the final states have electron configurations with two valence holes and an electron excited on a molecular or Rydberg orbital. Such final states are often dissociative, leading to the production of ionic and neutral fragments. In the third region, above $170.5 \mathrm{eV}$, an S 2p

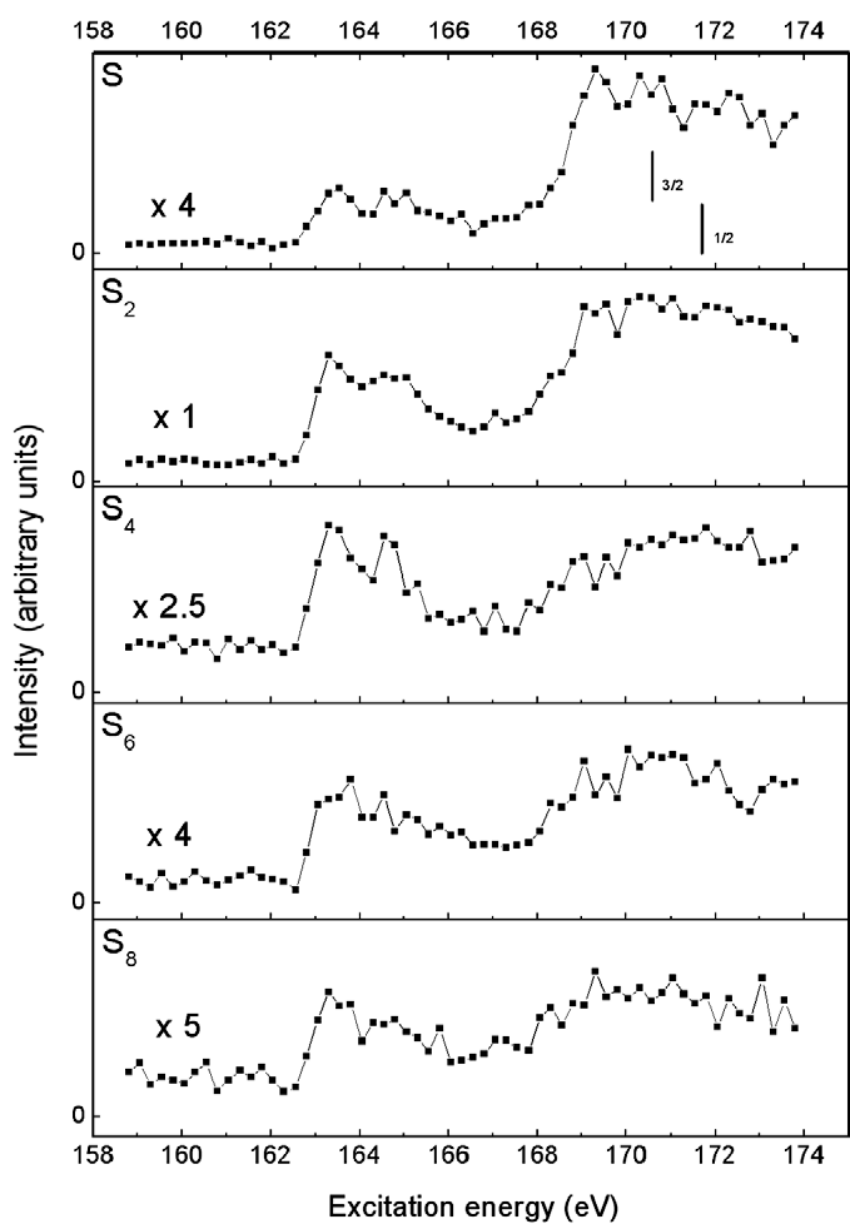

Fig. 4. Partial ion yield of different $S$ clusters across the $S 2 p$ edge. All spectra have been scaled to the most intense one, $S_{2}$. The $S 2 p$ ionization energies at 170.5 $\left(\mathrm{S} 2 \mathrm{p}_{3 / 2}\right)$ and $171.7 \mathrm{eV}\left(\mathrm{S} 2 \mathrm{p}_{1 / 2}\right)$ [18] are included as vertical lines.

electron can be ionized. The core hole is predominantly filled by Auger decay, which creates doubly charged final states. These states are typically dissociative, and two charged fragments may be produced [15].

After core excitation and core ionization, the expected outcome is therefore that the original neutral clusters break up into smaller fragments. In the spectrum measured above the $S 2 \mathrm{p}$ photoionization limits, the fragments $\mathrm{S}_{2}{ }^{+}$and $\mathrm{S}^{+}$have the highest peaks, while in the valence region (we tuned the synchrotron light to a photon energy of $21.2 \mathrm{eV}$ ) the presence of heavier cluster ions, especially those of $\mathrm{S}_{4}{ }^{+}$and $\mathrm{S}_{8}{ }^{+}$, is much more evident. Teodorescu et al. [15] studied the fragmentation of the sulfur clusters by measuring photoelectron-photoion photoion coincidence (PEPIPICO) spectra. Their results should be valid also for the present experimental conditions, so the reader is kindly advised to see Ref. [15] for further considerations on the fragmentation pathways of sulfur clusters.

\section{Conclusions}

In this work we have proved the feasibility of combining laser ablation and synchrotron radiation to generate and investigate clusters from solid samples. We have described in detail the experimental apparatus designed for that purpose and presented the first results. Sulfur clusters were created and their ionization across the $S 2$ p edge was studied yielding similar results to the ones obtained by heating, hence validating our method for producing clusters from laser ablated samples [15]. 
The designed apparatus is flexible enough to house other spectroscopic techniques (photon and electron detectors) which are planned to be mounted in the near future. Photoelectron-photoion coincidence experiments and time resolved synchrotron induced fluorescence spectroscopy are our next goal.

The presented experimental technique opens a wealth of possibilities for the study of clusters with a relatively simple set up which can mean an inflexion point in the field of free clusters. Moreover, the study of other species created in the ablation (radicals and short lived atoms/molecules) and the investigation of the laser ablation process itself are also possible and should be explored in future experiments.

\section{Acknowledgements}

J. Alvarez thanks CNR and the Short-Term-Mobility Program for travel and accommodation expenses. J. Alvarez and M. Sanz want also to thank CSIC-CAM support under the Project CCG07-CSIC/ ESP-2213 for travel and equipment. A.Casu acknowledges "Sardegna Ricerche" for funding his visit at INFM-TASC. A.M. Juarez-Reyes and L.M. Hoyos kindly acknowledge the support of ICTP. J. Alvarez and M. Stankiewicz thank EU support from VII Framework Program Transnational Access during the experiments at the Elettra Synchrotron Facility.

\section{References}

[1] E. Rühl, Int. J. Mass Spectrom. 229 (2003) 117.

[2] O. Björneholm, G. Öhrwall, M. Tchaplyguine, Nucl. Instrum. Meth. A 601 (2009) 161.

[3] M. Tchaplyguine, S. Peredkov, A. Rosso, I. Bradeanu, G. Öhrwall, S. Legendre, S. Sorensen, N. Mårtensson, S. Svensson, O. Björneholm, J. Electron Spectrosc. Relat. Phenom. 166-167 (2008) 38.

[4] P. Piseri, T. Mazza, G. Bongiorno, C. Lenardi, L. Ravagnan, F. Della Foglia, F. DiFonzo, M. Coreno, M. de Simone, K. Prince, P. Milani, New J. Phys. 8 (2006) 1.

[5] S. Orlando, A. Paladini, A. Santagata, V. Marotta, G.P. Parisi, M. Satta, D. Scuderi, D. Catone, A. Giardini, A. Mele, Int. J. Photoenergy 6 (2004) 23.

[6] M. Jadraque, J. Alvarez, R. de Nalda, M. Martin, Appl. Surf. Sci. 253 (2007) 6339.

[7] J. Alvarez-Ruiz, M. López-Arias, R. de Nalda, M. Martín, A. Arregui, L. Bañares, Appl. Phys. A 93 (2009) 681.

[8] T. Glover, G. Ackerman, R. Lee, D. Young, Appl. Phys. B 78 (2004) 995.

[9] C. Nicolas, J. Shu, D.S. Peterka, M. Hochlaf, L. Poisson, S.R. Leone, M. Ahmed, J. Am. Chem. Soc. 128 (2006) 220.

[10] L. Belau, S.E. Wheeler, B.W. Ticknor, M. Ahmed, S.R. Leone, W.D. Allen, H.F. Schaefer, M.A. Duncan, J. Am. Chem. Soc. 129 (2007) 10229

[11] M. Citir, R.B. Metz, L. Belau, M. Ahmed, J. Phys. Chem. A 112 (2008) 9584

[12] O. Kostko, M. Ahmed, R.B. Metz, J. Phys. Chem. A 113 (2009) 1225.

[13] K.C. Prince, R.R. Blyth, R. Delaunay, M. Zitnik, J. Krempasky, J. Slezak, R. Camilloni, L. Avaldi, M. Coreno, G. Stefani, C. Furlani, M. de Simone, S. Stranges, J. Synchrotron Radiat. 5 (1998) 565.

[14] J. Berkowitz, J.R. Marquart, J. Chem. Phys. 39 (1963) 275.

[15] C.M. Teodorescu, D. Gravel, E. Rühl, J. Chem. Phys. 109 (1998) 9280.

[16] A.K. Hearley, B.F.G. Johnson, J.S. McIndoe, D.G. Tuck, Inorg. Chim. Acta 334 (2002) 105

[17] N. Alberti, O. Šedo, J. Havel, Polyhedron 22 (2003) 2601.

[18] M.S. Banna, D.C. Frost, C.A. McDowell, B. Wallbank, Chem. Phys. Lett. 43 (1976) 426. 